\title{
Resposta cardiovascular na prova de esforço: pressão arterial sistólica
}

\author{
Luiz Carlos Passaro ${ }^{1}$
}

\section{INTRO DUÇÃO}

A ceita-se que em nível de $40 \%$ da capacidade de trabalho aeróbio o volume sistólico (VS) atinge seu máximo(23). Isso aplica-se tanto a indivíduos treinados, quanto destreinados, de ambos os sexos.

O VS máximo do indivíduo bem treinado é bem maior do que 0 de pessoa sedentária, graças à participação de fatores hereditários e à maior potência contrátil do miocárdio(4).

0 débito cardíaco $(\mathrm{DC})$, produto do VS pela freqüência cardíaca ( $F C)$, aumenta continuadamente com o exercício, mas o equilíbrio do DC a partir de 40 a $50 \%$ da capacidade máxima é mantido, basicamente, à custa da elevação da FC. A elevação do DC também será maior nos indivíduos treinados.

Embora exista vasodilatação periférica que se estabelece durante a atividade física, ocorre elevação da pressão arterial sistólica (PAS) devido fundamentalmente ao incremento do DC.

\section{ASPECTOS FISIOLÓGICOS DA PRESSÃO ARTERIAL SISTÓLICA NO EXERCÍCIO}

Em 1889 já se demonstrava que a PAS e a FC se elevavam durante e imediatamente após o trabalho muscular ${ }^{(11)}$. Em geral, essa elevação da PAS é acompanhada de redução ou nenhuma modificação na pressão arterial diastólica (PAD).

A pressão sanguínea na aorta é mantida pela integração dos seguintes fatores: DC, resistência periférica, elasticidade das principais artérias, viscosidade do sangue e volume sanguíneo.

O fluxo sanguíneo local é determinado principalmente por uma cabeça de pressão e pelo diâmetro dos vasos presentes. 0 tônus vasomotor é fornecido pelas fibras simpáticas vasoconstritoras provenientes da área vasomotora na medula oblonga; a substância transmissora é a noradrenalina. Os receptores de membrana do tipo alfa têm distribuição comum no leito vascular e promovem vasoconstrição. Existem receptores do tipo beta em algumas seções précapilares de resistência, tais como nos músculos esqueléti-

1. Cardiologista da Fitcor. Diretor de Publicações do Departamento de Ergometria e Reabilitação Cardiovascular da Sociedade Brasileira de Cardiologia. (Fonte: Centro de Estudos da Fitcor - SP). cos e no miocárdio, onde a adrenalina pode levar ao relaxamento dos músculos lisos destes vasos(6).

Existe incremento mais acentuado da PAS nos dois primeiros minutos de exercício (fase de aquecimento); após o aquecimento, se estabelece um valor constante (fase de equilíbrio) e o nível da PAS a seguir dependerá da intensidade do exercício.

Quando o trabalho físico é interrompido, ocorre queda imediata da pressão arterial (PA $)^{(2)}$. Na fase de recuperação, observa-se diminuição mais ou menos uniforme da PA, exceto no intervalo entre o segundo e o quarto minuto, quando há menor redução dos valores percentuais ${ }^{(29)}$.

A extensão da queda de PA dependerá da postura, da temperatura ambiente, da duração do exercício e da interrupção súbita ou não do exercício. A PA eleva-se pouco acima dos níveis prévios ao esforço.

A partir de dados obtidos da literatura têm-se os valores médios de PAS observados no quadro 1 para adultos jovens do sexo masculino(2).

\section{QUADRO 1}

\begin{tabular}{|c|c|c|c|c|c|}
\hline \multirow{2}{*}{$\begin{array}{l}\text { Função } \\
\text { PAS }\end{array}$} & \multicolumn{2}{|c|}{ Repouso } & \multicolumn{2}{|c|}{ Exercício moderado } & \multirow{2}{*}{$\frac{\text { Exercício máximo }}{\text { Em pé }}$} \\
\hline & Supino & Em pé & Supino & Em pé & \\
\hline $\mathrm{mmHg}$ & 120 & 130 & 165 & 175 & 215 \\
\hline
\end{tabular}

Os exercícios realizados com os braços elevam mais significativamente a PA do que 0 trabalho de pernas ${ }^{(5)}$. A PA é relativamente alta em exercícios com pequenos grupos musculares e é provável que isto seja decorrente de vasoconstrição nos músculos inativos.

Quanto maiores forem os grupos musculares ativados, mais pronunciada será a dilatação dos vasos de resistência. A resistência periférica mais baixa é refletida em PA mais baixa. Também se observa maior elevação da PAS na execução de exercício isométrico do que no exercício isotônico. No exercício isométrico ou estático no qual a contração muscular é mantida, a resposta de dilatação das arteríolas no interior do músculo é impedida pela compressão mecânica da vasculatura no interior do músculo que permanece em contração. Como conseqüência, a combinação de aumento do DC sem redução da resistência vascular sistêmica 
produz maior elevação da PAS e da pressão arterial diastólica (PAD)(17).

Em esforços leves e moderados a PAS atinge estado de equilíbrio após alguns minutos e, segundo Hollmann, em esforços mais intensos a PAS aumenta até o final do exercício(24). Os limites fisiológicos durante esforços máximos ainda não estão totalmente definidos, podendo observar-se valores de PAS superiores a $250 \mathrm{mmH} g$ em atletas em treinamento básico de resistência.

A reserva sistólica de PA (RS), isto é, o valor diferencial entre a PAS do esforço máximo e a PAS de repouso, pode evidenciar o rendimento cardíaco de pressão(24). Assim, pessoas treinadas apresentam RS de 100 a $150 \mathrm{mmH} \mathrm{g;} \mathrm{seden-}$ tários normais, de 75 a $100 \mathrm{mmH}$ g; e cardiopatas, valores inferiores a $75 \mathrm{mmH}$. A RS também está algo reduzida em hipertensos.

Admite-se que a PAS deva aumentar cerca de $7,3 \mathrm{mmH} \mathrm{g}$ por equivalente metabólico (MET) como média de esforço e até um máximo de $15 \mathrm{mmH} g^{(27)}$. Recentemente, foram observadas em indivíduos jovens do sexo masculino variações de 5 a $6 \mathrm{mmH}$ g por MET (Naughton J L: Comunicação pessoal, 1981); valores semelhantes foram descritos em nosso meio(3). Níveis superiores caracterizariam a hipertensão reativa ao esforço, que pode ser um sinal premonitório da hipertensão arterial.

Em nosso serviço temos desenvolvido curvas de PA em população paulista saudável, sedentária, de ambos os se$x{ }^{(28)}$. As faixas etárias foram separadas em grupos divididos a cada cinco anos, considerando-se normais os valores contidos na média e dois desvios-padrões.

A PAS durante o esforço físico é mais elevada nos indivíduos idosos. Esse fato pode ser comprovado em estudo que desenvolvemos com 80 indivíduos hígidos de diferentes faixas etárias, em teste de esforço em esteira rolante(21). Também em cicloergômetro esse comportamento da PAS pôde ser evidenciado(29).

O s homens geralmente apresentam em cada nível de esforço submáximo FC mais baixa do que as mulheres. No entanto, a FC máxima em ambos os sexos não parece diferir. A FC mais baixa do homem associa-se a maior volume sistólico, fato este que se deve ao maior tamanho do coração do homem, maior volume sanguíneo e, em geral, maior nível de condicionamento físico ${ }^{(30)}$. A o contrário do que ocorre com a FC, parece que a PA durante o esforço em homens e mulheres se comporta de forma semelhante(24); o retorno aos níveis de repouso após o exercício se dá de forma mais rápida nos homens.

Por outro lado, na experiência de Alfieri et al.(1), de modo geral, as mulheres apresentam variações da PAS sensivelmente inferiores durante 0 exercício, em relação aos homens. Níveis fixos e até queda da PAS podem observar-se em mulheres hígidas. Em nosso laboratório temos observado tendência semelhante(28).

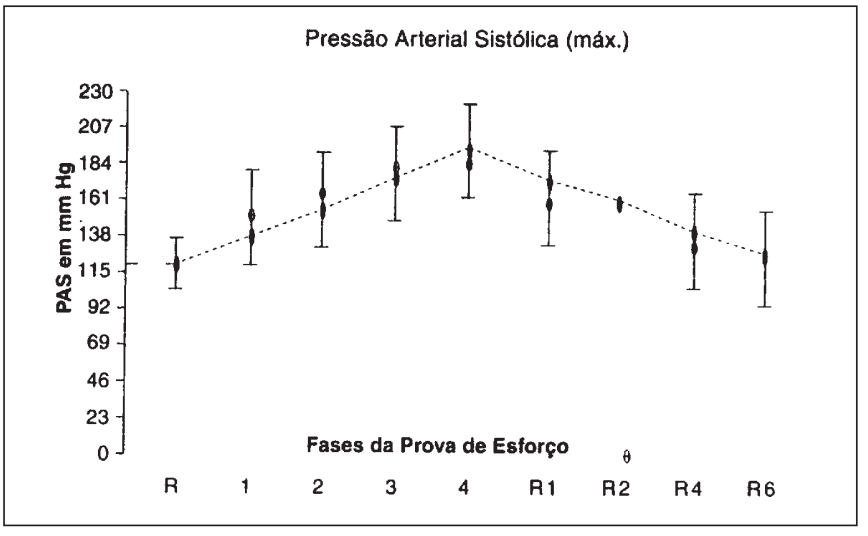

Fig. 1 - Curva fisiológica da PAS durante a prova de esforço

Em relação ao treinamento físico, os indivíduos treinados têm PAS de repouso baixa e os atletas de resistência bem treinados atingem o estado de equilíbrio da PAS mais rapidamente com grau de elevação total mais baixo ${ }^{(24)}$.

\section{CONSIDERAÇÕES METODOLÓGICAS NA AFERIÇÃO DA PAS}

É sabido que a obtenção da PA durante a prova de esforço não é tarefa fácil e a dificuldade é ainda maior quando se trata da obtenção da PAD e, sobretudo, quando o ergômetro utilizado é a esteira rolante.

Em análise comparativa de PAS avaliada por aferição direta intra-arterial e através da ausculta dos sons de Korotkoff, não se evidenciaram diferenças significativas no repouso supino. Q uando os indivíduos caminharam, de pé, em várias velocidades e diferentes inclinações da rampa, o método auscultatório subestimou a pressão de 5,1 a 10,3\% (8 a $15 \mathrm{mmH} \mathrm{g}$ ). Maiores foram as diferenças após o exercício(18).

Irving et al.(19) concluíram que a acurácia da esfigmomanometria foi satisfatória em $75 \%$ das pessoas estudadas. Clausen e Trap-J ensen ${ }^{(10)}$ não evidenciaram diferenças significativas entre a PAS intra-arterial e a aferida pelo método auscultatório durante exercício em cicloergômetro. Esses dados positivos citados para a PAS não se aplicaram aos estudos da aferição de PAD.

Carvalho et al. ${ }^{(9)}$, em prova de esforço de pacientes anginosos, utilizando cicloergômetro adaptado à realização simultânea de estudo hemodinâmico invasivo em posição supina, demonstraram correlação significativa $(r=0,76)$ entre a PAS medida pelo cateterismo cardíaco e pela esfigmomanometria.

Foi demonstrada boa reprodutibilidade da curva de PAS durante o exercício em esteira rolante, com diferença média não significativa de $8,6 \mathrm{mmH} \mathrm{g}^{(19)}$.

\section{RESPOSTA ANÔMALA DA PAS AO EXERCíCIO}

A elevação exagerada da PAS durante o exercício no indivíduo com PA normal na fase de repouso, a chamada res- 


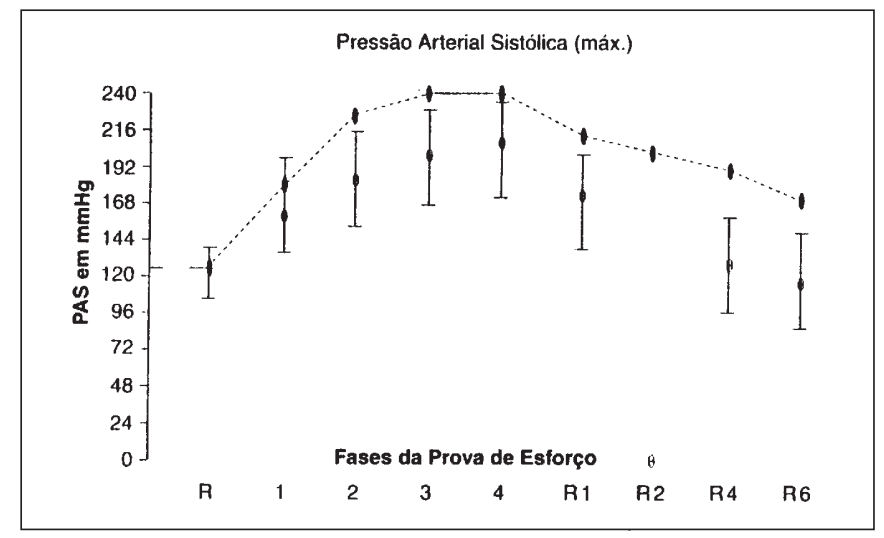

Fig. 2 - Curva da PAS com resposta do tipo "hiper-reativa"

posta "hiper-reativa" da PA ("HR"), pode ser um sinal premonitório da hipertensão arterial(12,25,31).

A figura 2 mostra uma resposta "HR" de PAS, com valor superior ao da média mais dois desvios-padrões quando confrontado com os da curva normal. Por outro lado, a resposta deprimida da PAS pode ter várias etiologias (quadro 2); contudo, a doença coronariana obstrutiva é a etiologia mais comum desta resposta pressórica(11).

\section{QUADRO 2}

1. Doença arterial coronariana

2. Exercício vigoroso prolongado

3. Insuficiência cardíaca

4. Taqui ou bradicardia

5. Estenose ou insuficiência aórtica

6. Estenose ou insuficiência mitral

7. Coarctação da aorta

8. "Shunt" intracardíaco

9. Hipovolemia

10. Tono vasomotor anormal

11. Cardiomiopatia obstrutiva

12. Drogas

Em estudo de 1.586 homens com clínica de insuficiência coronariana concluiu-se que a PAS máxima de esforço é melhor parâmetro prognóstico de mortalidade do que o segmento ST. A correlação da resposta sistólica ao esforço e a taxa anual de mortalidade por 1.000 analisada nesse estudo foi: PAS < $140 \mathrm{mmHg} 97,0$; PAS $140-199 \mathrm{mmHg} 25,3 \mathrm{e}$ PAS $>200 \mathrm{mmHg} 6,6^{(19)}$. O utro estudo abrangendo 1.852 pacientes masculinos também demonstrou ser a PAS máxima no exercício um dos melhores parâmetros prognósticos de mortalidade cardiovascular ${ }^{(8)}$.

Ellestad et al. ${ }^{(14)}$, estudando 215 pacientes com teste positivo (125 verdadeiros-positivos e 95 falsos-positivos), demonstraram que a resposta sistólica foi a segunda melhor variável para a correta classificação do teste de esforço.

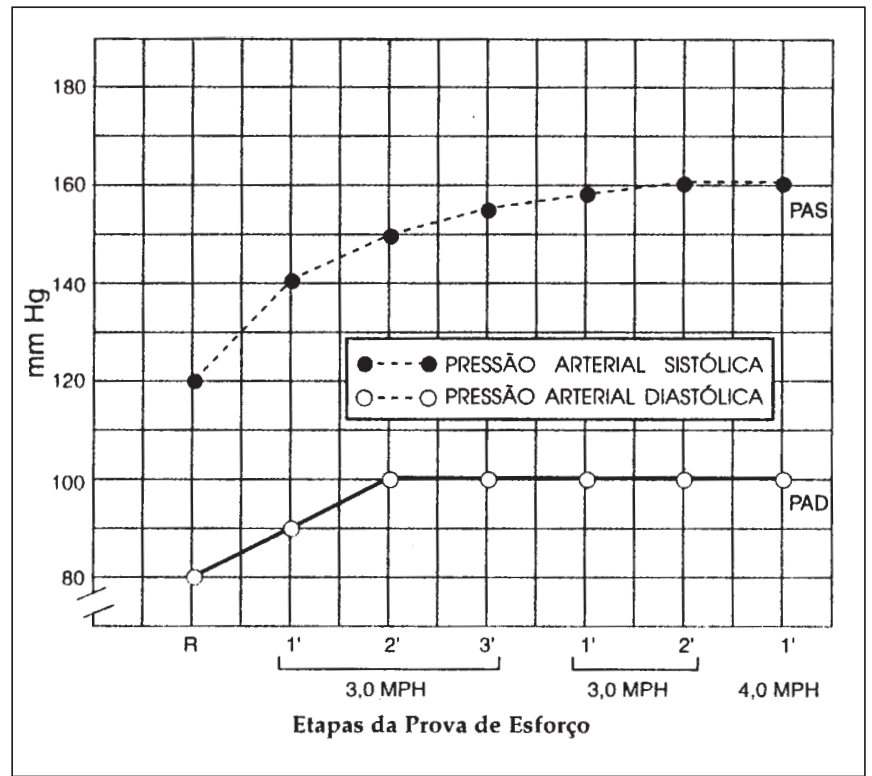

Fig. 3 - PacienteW.F.W., 38 anos, masc., que mostrou curva de pressão arterial sistólica deprimida, em teste de esforço de protocolo máximo, em esteira rolante

M orris et al.(26), estudando 22 pacientes com lesões coronárias obstrutivas superiores a $75 \%$, evidenciaram hipotensão esforço-induzida em sete destes pacientes. Em seis pacientes que repetiram a prova de esforço após revascularização miocárdica houve normalização da resposta de PAS.

Godoy et al. ${ }^{(15)}$ demonstraram a diferença da resposta sistólica entre indivíduos normais e coronariopatas estudando a diferença da PAS de repouso e esforço máximo. Esse valor foi significativamente distinto entre anginosos (valores mais baixos), infartados (valores intermediários) e normais (valores mais altos).

Em nosso serviço a curva de PA no teste de esforço é bastante valorizada, com protocolo que exige aferições a

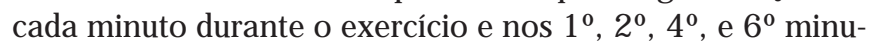
tos da fase de recuperação.

Alguns critérios devem ser seguidos para a obtenção de uma curva pressórica confiável. Procuramos suspender as drogas adequadamente, sempre um mesmo observador e a mesma posição de tomada da PA, execução de pelo menos três estágios de um protocolo clássico, uso de esfigmomanômetro de coluna de mercúrio e, se possível, continuar o esforço por pelo menos mais um estágio para configurar a queda de PA ${ }^{(16)}$.

Em nosso laboratório procuramos caracterizar o tipo de resposta de PA quando esta resulta de adaptação inadequada do ventrículo esquerdo ao exercício. Pode-se observar incremento uniforme mas achatado da PAS durante todo 0 exercício, caracterizando uma curva deprimida (fig. 3); incremento proporcional no início e estabilização da PAS apesar da progressão do trabalho físico, obtendo-se uma curva 


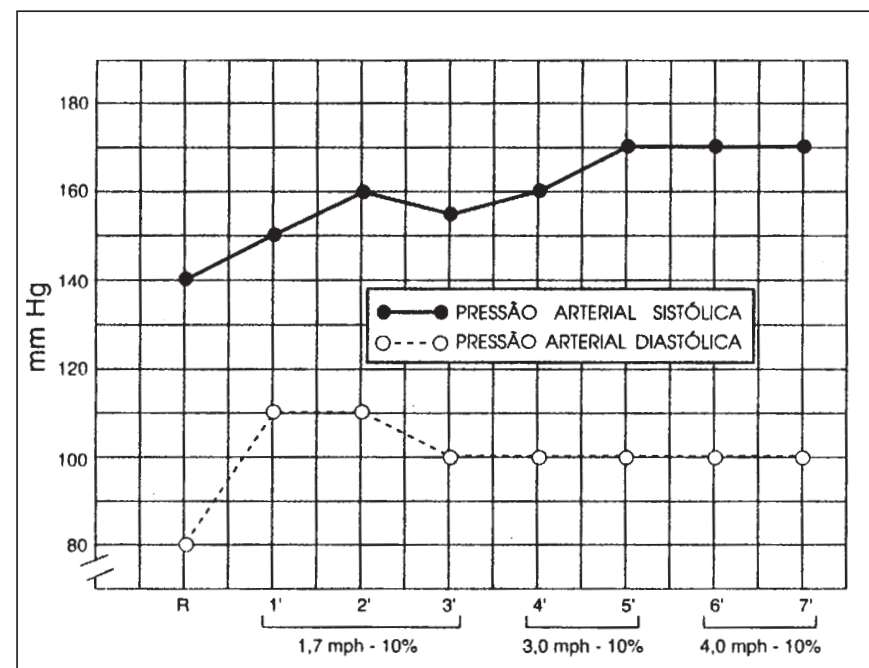

Etapas da Prova de Esforço

Fig. 4 - Pressão arterial sistólica deprimida, em platô, no paciente R.A.L., de 38 anos, masc., com infarto do miocárdio prévio

deprimida em platô (fig. 4); incremento inicial e queda com a progressão do exercício (fig. 5).

Acreditamos que, para tomar uma disfunção ventricular como decorrente da isquemia coronariana, devem-se comparar os dados obtidos da curva de PAS do paciente com os obtidos no mesmo ergômetro e no grupo equivalente e mesma faixa etária. Embora tenhamos defendido há alguns anos a diferença da PA (PAS do momento de pico menos a PAS da fase de repouso) como indicadora de disfunção ventricular, esse dado isolado não parece suficiente para estabelecer tal diagnóstico.

Para tal torna-se necessária a análise global das respostas da PAS e PAD. Q uando a queda da PAS é acompanhada de redução da PAD, a disfunção ventricular é improvável, pois esta se acompanha de PAD estável ou aumentada, em razão do mecanismo de compensação (aumento da resistência periférica).

A elevação paradoxal da PAS logo após a cessação do esforço sugere resposta inadequada do ventrículo esquerdo, cuja fisiopatologia é compatível com isquemia miocárdica (fig. 6).

Comentando a resposta de manutenção da PAS sem queda na fase de recuperação do teste de esforço, tida como resposta possivelmente relacionada à isquemia miocárdica, Ellestad ${ }^{(13)}$ questiona essa possibilidade, discutindo a dificuldade de obtenção da PA em cargas altas de esforço.

O utra causa que pode levar a comportamento deprimido da PAS no exercício é a doença de Chagas, mesmo em sua forma indeterminada. Essa doença reveste-se de importância, particularmente em nosso meio.

Existem dados em nossa literatura evidenciando a resposta inotrópica deprimida na doença de Chagas, em que tam-

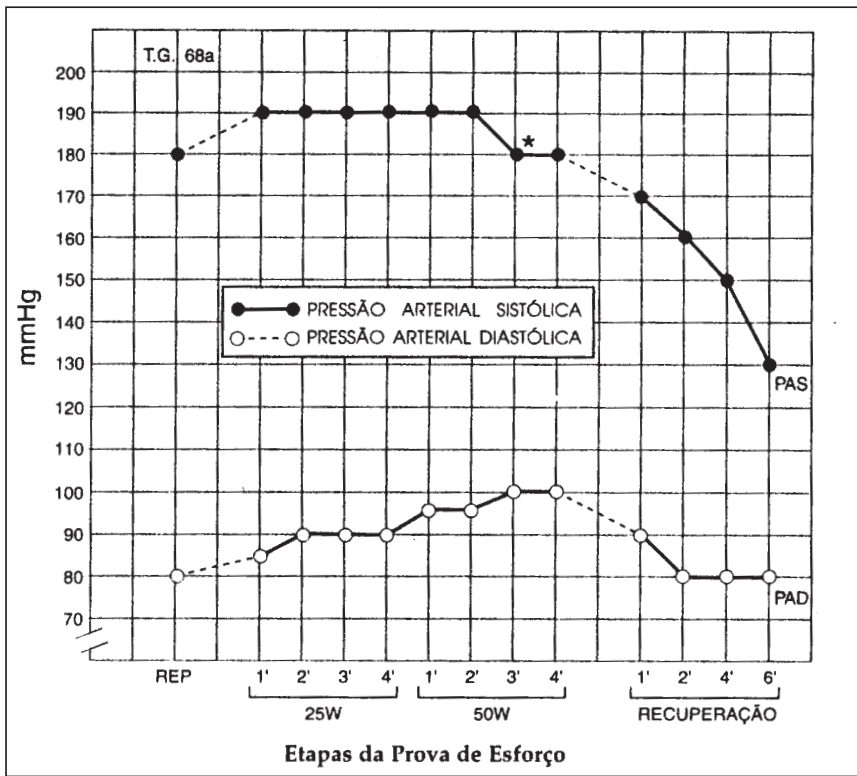

Fig. 5 - Paciente T.G., de 68 anos, portador de doença coronária obstrutiva, com queda da pressão arterial sistólica simultânea à elevação da pressão arterial diastólica, no terceiro minuto de 50 watts, concomitante à manifestação de angina típica

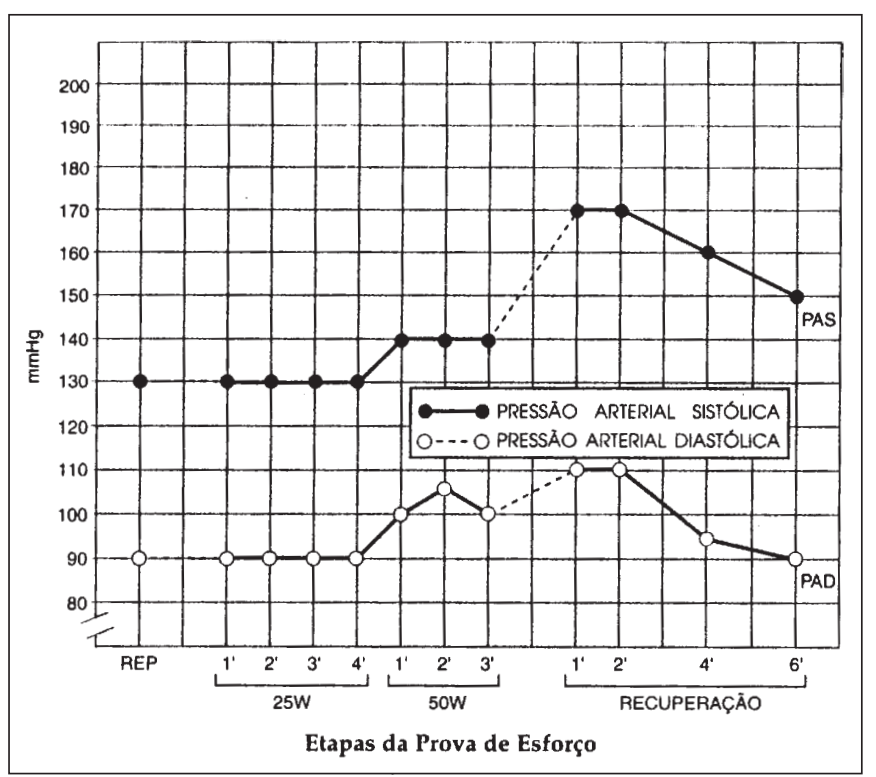

Fig. 6 - Resposta deprimida da pressão arterial sistólica em exercício com elevação paradoxal das pressões arteriais sistólica e diastólica na fase de recuperação, em paciente com doença coronária obstrutiva

bém é discutida a elevação da PAD ao esforço como um mecanismo de adaptação cardiovascular ${ }^{(7,22)}$.

\section{CONCLUSÃO}

É possível a definição de um padrão normal da resposta da PAS ao exercício e ainda agrupá-la em diferentes faixas etárias. A busca de correta curva de PA deve ser objetivo da maior importância. 
A avaliação cuidadosa dos parâmetros cardiovasculares em exercício é fundamental para que se tenham para cada faixa etária os valores médios populacionais.

Embora as pressões arteriais verificadas durante a prova de esforço possam trazer dificuldades de interpretação, equipe com experiência é capaz de julgá-las com boa precisão. J ulgamos que os sons de Korotkoff sejam mais facilmente perceptíveis durante o exercício do que na fase de repouso, em função da maior amplitude da onda de fluxo sanguíneo.

A curva de PA será tanto mais fiel quanto maior for 0 número de medidas; portanto, uma prova de esforço de curta duração não permite 0 apoio suficiente para o estabelecimento do diagnóstico de disfunção ventricular.

Salienta-se que, embora não deva ser excluída a valorização das quedas isoladas da PAS, elas deverão ser cuidadosamente avaliadas e associadas a outros parâmetros mais seguros para definição da PAS deprimida em exercício.

Assim, a associação entre as respostas cardiovascular, clínica e eletrocardiográfica faz da prova de esforço um instrumento cada vez mais útil ao cardiologista.

\section{REFERÊNCIAS}

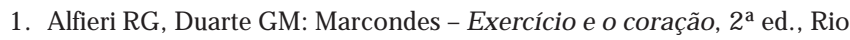
de J aneiro, Editora Cultura Médica, 1993, 95p.

2. Andersen KL, Shephard RJ, Denolin H, Varnauskas E, Masironi R: Fundamentals of exercise testing, World H ealth Organization, Geneva, 1971.

3. A raujo WB: Ergometria e cardiologia esportiva, Rio de J aneiro, MEDSI, Editora Médica e Científica Ltda., 1986, 139p.

4. Astrand PO, Cuddy TE, Saltin B, Stienberg J : Cardiac output during submaximal and maximal work. J Appl Physiol 19: 268, 1964.

5. Astrand PO, Ekblom B, Messin R, Saltin B, Stenberg J : Intra-arterial blood pressure during exercise with different muscle groups. J Appl Physiol 20: 253, 1965.

6. Astrand PO, Rodahl K: Tratado de fisiologia do exercício, Rio de J aneiro, Editora Guanabara S.A., 1987, p. 145.

7. Bellini AJ , Nicolau J C, Bilaqui A: Prova de esforço na forma subclínica da doença de Chagas. Arq Bras Cardiol 33 (Suppl 2): 261, 1977.

8. Bruce RA, Derowen T, Peterson DR et al: Non-invasive predictors of sudden cardiac death in men with coronary heart disease: predictive value of maximal stress testing. Am J Cardiol 39: 833-840, 1977.

9. Carvalho VB, Passaro LC, Décourt LV et al: A lterações eletrocardiográficas durante arteriografia coronária. Arq Bras Cardiol 41: 80, 1983.

10. Clausen J P, Trap-J ensen J : H eart rate and arterial blood pressure during exercise in patients with angina pectoris. Circulation 53: 436-442, 1976.

11. Comess KA, Fenster PE: Clinical implications of the blood pressure reponse to exercise. Circulation 68: 233-244, 1981.

12. Dlin RA et al: Follow up of normotensive men with exaggerated blood pressure response to exercise. Am Heart J 106: 316-320, 1983.

13. Ellestad MH: Stress testing: principles and practice, Philadelphia, F.A. Davis Company, 1986.
14. Ellestad MH, Savitz S, Bergdau D, Teske J : The false positive stress test: multivariate analysis of 215 subjects with hemodynamic angiographic and clinical date. Am J Cardiol 40: 681-685, 1977.

15. Godoy et al: Dados de eletrocardiografia de esforço: análise da curva de pressão arterial sistólica. In Del Nero J unior E, Papaléo Netto M, M offa P, O rtiz J (eds.): Semiologia cardiológica não-invasiva, Rio de J aneiro, Epume, 1979, 530.

16. Godoy M, Muto RT, Passaro LC et al: Teste de esforço na avaliação da insuficiência coronária. In Carvalho VB, Macruz R (eds.): Cardiopatia isquemica: aspectos de importância clínica, São Paulo, Sarvier 1989, 292.

17. Hanson P, Nagle F: Exercício isométrico: respostas cardiovasculares nas populações normal e cardíaca. In Hasson P (ed.): Clínicas cardiológicas, Rio de J aneiro, Interlivros Edições Ltda., 1987, p. 159-172.

18. Henschel A, Vega F. de la, Taylor HL: Simultaneous direct and indirect blood pressure measurements in man at rest and work. J Appl Physiol 6: 506-508, 1954

19. Irving J B, Bruce RA, Derowen TA: Variations in and significance of systolic pressure during maximal exercise (treadmill) testing: relation to severity of coronary artery disease and cardiac mortality. Am J Cardiol 39: 841-848, 1977.

20. J ackson AS et al: Prediction of future resting hypertension from exercise blood pressure. J Cardiac Rehab 3: 263, 1983.

21. Magliano MFM, Passaro LC, J arussi FA, Godoy M: A nálise das pressões arteriais, freqüência cardíaca, duração do teste e outros parâmetros, em indivíduos normais, submetidos à ergometria em esteira rolante. Arq Bras Cardiol 37 (Supl 1): 97, 1981.

22. Marins N, Domenico $M$, Lobo $C$ et al: Comportamento anômalo da pressão arterial sistólica (PAS) durante o teste ergométrico; contribuição ao diagnóstico da miocardiopatia. Arq Bras Cardiol 32 (Suppl 1): 131, 1976.

23. Mathews DKI, Fox EL: Bases fisiológicas da educação física e dos desportos, 2 a ed., Rio de J aneiro, Editora Interamericana Ltda., 1979, $123 p$.

24. Mellerowicz H : Ergometria, 3a ed., Buenos Aires, Editorial Médica Panamericana S.A., 1984, p. 110-111.

25. Miller-Craig $M$ et al: $U$ se of graded exercise testing in assessing the hypertensive patient. Clin Cardiol 3: 236-240, 1980.

26. Morris SN, Phillips J F, J ordan J W, MCH enry PL: Incidence and significance of decreases in systolic blood pressure during gradual treadmill exercise testing. AmJ Cardiol 41: 221-226, 1978.

27. Naughton J, Haider R: Methods of exercise testing in exercise testing and exercise training. In Naughton J, H ellerstein HK (eds.): Coronary heart disease, New York, Academic Press, 1969.

28. Solórzano J A, Godoy M, Escobar CLD et al: Valores populacionais dos índices de resposta cardiovascular obtidos do teste de esforço em esteira rolante. Arq Bras Cardiol 49: 127, 1987.

29. Solórzano J A, Godoy M, Morán CAM et al: A nálise da freqüência cardíaca, pressões arteriais e índice tensão/ tempo em exercício. Arq Bras Cardiol 31: 256, 1978.

30. Wilmore J H, Thomas EL: Importância das diferenças entre o homem e a mulher na prova de esforço e na prescrição de exercícios. In Skinner J S (ed.): Prova de esforço e prescrição de exercícios para casos específicos, Rio de J aneiro, Livraria e Editora Revinter, 1991, p. 35-53.

31. Wilson NV, Meyer BM: Early prediction of hypertension using exercise blood pressure. Prev Med 10: 62-68, 1981. 